\title{
A gauge invariant dressed holon and spinon description of the normal-state of underdoped cuprates
}

\author{
Shiping Feng, Jihong Qin, and Tianxing Ma \\ Department of Physics, Beijing Normal University, Beijing 100875, China
}

\begin{abstract}
A partial charge-spin separation fermion-spin theory is developed to study the normal-state properties of the underdoped cuprates. In this approach, the physical electron is decoupled as a gauge invariant dressed holon and spinon, with the dressed holon behaving like a spinful fermion, representing the charge degree of freedom together with the phase part of the spin degree of freedom, while the dressed spinon is a hard-core boson, representing the amplitude part of the spin degree of freedom. The electron local constraint for the single occupancy is satisfied. Within this approach, the charge and spin dynamics of the underdoped cuprates are studied based on the $t-t^{\prime}-J$ model. It is shown that the charge dynamics is mainly governed by the scattering from the dressed holons due to the dressed spinon fluctuation, while the scattering from the dressed spinons due to the dressed holon fluctuation dominates the spin dynamics.
\end{abstract}

74.25.Fy, 74.25.Ha, 74.72.-h

\section{INTRODUCTION}

After over fifteen years of intense experimental studies of doped cuprate superconductors, a significant body of reliable and reproducible data has been accumulated by using many probes ${ }^{1,2}$, which shows that the most remarkable expression of the nonconventional physics is found in the normal-state ${ }^{1,2}$. The normal-state properties exhibit a number of anomalous properties in the sense that they do not fit in the conventional Fermiliquid (FL) theory ${ }^{2-4}$, and are closely related to the fact that these materials are doped Mott insulators ${ }^{1-4}$. The ground state in the undoped case is an antiferromagnetic (AF) long-range-order (AFLRO) Néel state, but changing the carrier concentration by ionic substitution or increasing the oxygen content turns these compounds into strongly correlated metals leaving the AF short-rangeorder correlation still intact ${ }^{1,2}$. It is then not surprising that the nonconventional behaviors are most striking in the underdoped regime, where the concentration of doped holes is small. The anomalous properties observed in a variety of experiments, such as the nuclear magnetic resonance (NMR), nuclear quadrupole resonance (NQR), muon spin rotation $(\mu S R)$ techniques, inelastic neutron scattering studies ${ }^{5-10}$, optical and transport measurements ${ }^{11^{-14}}$, and angle-resolved photoemission spectroscopy (ARPES) investigation ${ }^{15}$, exclude conventional theories. The single common feature in doped cuprates is the two-dimensional $(2 \mathrm{D}) \mathrm{CuO}_{2}$ plane $^{1,2}$, and it seems evident that the nonconventional behaviors are dominated by this plane. Very soon after the discovery of high-temperature superconductivity (HTSC) in doped cuprates, Anderson proposed a scenario of HTSC based on the charge-spin separation (CSS) in $2 \mathrm{D}^{3}$, where the internal degrees of freedom of the electron are decoupled as the charge and spin degrees of freedom, while the elementary excitations are not quasi-particles but collective modes for the charge and spin degrees of freedom, i.e., the holon and spinon, then these holon and spinon might be responsible for the nonconventional behaviors. Many unusual properties of the underdoped cuprates are extensively studied following this line within the $2 \mathrm{D} t-J$ type model $^{4,16}$.

The decoupling of the charge and spin degrees of freedom of electron is undoubtedly correct in onedimensional (1D) interacting electron systems ${ }^{17}$, where the charge and spin degrees of freedom are represented by boson operators that describe the excitations of chargedensity wave and spin-density wave, respectively. In particular, the typical behavior of the non-FL, i.e., the absence of the quasiparticle propagation and CSS, has been demonstrated theoretically within the $1 \mathrm{D} t-J$ model $^{18}$. Moreover, the holon and spinon as the real elementary excitations in $1 \mathrm{D}$ cuprates have been observed directly by the ARPES experiment ${ }^{19}$. Therefore both theoretical and experimental studies indicate that the existence of the real holon and spinon is common in $1 \mathrm{D}^{20}$. However, the case in $2 \mathrm{D}$ is very complex since there are many competing degrees of freedom ${ }^{1,2}$. As a consequence, both experimental investigation and theoretical understanding are extremely difficult ${ }^{1-4}$. Among the unusual properties of the underdoped cuprates, a hallmark is the charge transport ${ }^{11-14}$, where the conductivity shows a non-Drude behavior at low energies, and is carried by $x$ holes, where $x$ is the hole doping concentration, while the resistivity exhibits a linear temperature behavior over a wide range of temperatures. This is a strong experimental evidence supporting the notion of CSS, since not even conventional electron-electron scattering would show the striking linear rise of scattering rate above the Debye frequency, and if there is no CSS, the phonons should affect these properties ${ }^{21}$. Moreover, it has been argued that the most plausible source of the absence of phonon scattering and of pair-breaking effects seems to be $\mathrm{CSS}^{21}$, and further, a compelling evidence for CSS in doped cuprates has been found from the experimental test of the Wiedemann-Franz law, where a clear departure from the universal Wiedemann-Franz law for the typical FL behavior is observed ${ }^{22}$. On the numerical study front, the crossover from the FL to non-FL behavior with decreas- 
ing the hole doping concentration near the Mott transition has been found within the $2 \mathrm{D} t-J$ model $^{23}$. Furthermore, it has been shown within the $2 \mathrm{D} t-t^{\prime}-J$ model by using the exact diagonalization method that there is a tendency of holes to generate nontrivial spin environments, this effect leads to a decoupling of the spin from charge $^{24}$. In this case, a formal theory with the gauge invariant holon and spinon, i.e., the issue of whether the holon and spinon are real, is centrally important ${ }^{25}$. In this paper, we propose a partial CSS fermion-spin theory, and show that if the local single occupancy constraint is treated properly, then the physical electron can be decoupled completely by introducing the dressed holon and spinon. These dressed holon and spinon are gauge invariant, i.e., they are real in 2D. As an application of this theory, we discuss the charge and spin dynamics of the underdoped cuprates within the $t-t^{\prime}-J$ model, and the results are qualitatively similar to that seen in experiments.

The paper is organized as follows. The framework of the partial CSS fermion-spin theory is presented in Sec. II. Within this theory, the single-particle dressed holon and spinon Green's functions of the $t-t^{\prime}-J$ model are calculated in Sec. III by considering the dressed holonspinon interaction, where the dressed holon and spinon self-energies are obtained by using the equation of motion method. In Sec. IV, we discuss the charge transport of the underdoped cuprates. The incommensurate (IC) spin response of the underdoped cuprates is studied in Sec. V. Sec. VI is devoted to a summary and discussions.

\section{GAUGE INVARIANT DRESSED HOLON AND SPINON}

We start from the $t-t^{\prime}-J$ model defined on a square lattice as $^{3,26}$,

$$
\begin{aligned}
H & =-t \sum_{i \hat{\eta} \sigma} C_{i \sigma}^{\dagger} C_{i+\hat{\eta} \sigma}+t^{\prime} \sum_{i \hat{\tau} \sigma} C_{i \sigma}^{\dagger} C_{i+\hat{\tau} \sigma} \\
& +\mu \sum_{i \sigma} C_{i \sigma}^{\dagger} C_{i \sigma}+J \sum_{i \hat{\eta}} \mathbf{S}_{i} \cdot \mathbf{S}_{i+\hat{\eta}}
\end{aligned}
$$

where $\hat{\eta}= \pm \hat{x}, \pm \hat{y}, \hat{\tau}= \pm \hat{x} \pm \hat{y}, C_{i \sigma}^{\dagger}\left(C_{i \sigma}\right)$ is the electron creation (annihilation) operator, $\mathbf{S}_{i}=C_{i}^{\dagger} \vec{\sigma} C_{i} / 2$ is spin operator with $\vec{\sigma}=\left(\sigma_{x}, \sigma_{y}, \sigma_{z}\right)$ as Pauli matrices, and $\mu$ is the chemical potential. The $t-t^{\prime}-J$ model (1) is subject to an important local constraint $\sum_{\sigma} C_{i \sigma}^{\dagger} C_{i \sigma} \leq 1$ that a given site can not be occupied by more than one electron. In the $t-t^{\prime}-J$ model, the strong electron correlation manifests itself by this constraint, and therefore the crucial requirement is to impose this constraint ${ }^{3,26-28}$. It has been shown that this constraint can be treated properly within the fermion-spin theory ${ }^{29}$. In this section, we show that the dressed holon and spinon in the fermion-spin theory are gauge invariant. Following the discussions in [29], we decouple the constrained electron operator as,

$$
C_{i \sigma}=h_{i}^{\dagger} a_{i \sigma},
$$

with the constraint $\sum_{\sigma} a_{i \sigma}^{\dagger} a_{i \sigma}=1$, where the spinless fermion operator $h_{i}$ keeps track of the charge degree of freedom, while the boson operator $a_{i \sigma}$ keeps track of the spin degree of freedom, then the Hamiltonian (1) can be rewritten as,

$$
\begin{aligned}
H & =-t \sum_{i \hat{\eta} \sigma} h_{i} a_{i \sigma}^{\dagger} h_{i+\hat{\eta}}^{\dagger} a_{i+\hat{\eta} \sigma}+t^{\prime} \sum_{i \hat{\tau} \sigma} h_{i} a_{i \sigma}^{\dagger} h_{i+\hat{\tau}}^{\dagger} a_{i+\hat{\tau} \sigma} \\
& -\mu \sum_{i} h_{i}^{\dagger} h_{i}+J \sum_{i \hat{\eta}}\left(h_{i} h_{i}^{\dagger}\right) \mathbf{S}_{i} \cdot \mathbf{S}_{i+\hat{\eta}}\left(h_{i+\hat{\eta}} h_{i+\hat{\eta}}^{\dagger}\right),
\end{aligned}
$$

where the pseudospin operator $\mathbf{S}_{i}=a_{i}^{\dagger} \vec{\sigma} a_{i} / 2$. In this case, the electron constraint $\sum_{\sigma} C_{i \sigma}^{\dagger} C_{i \sigma}=1-h_{i}^{\dagger} h_{i} \leq 1$ is exactly satisfied, with $n_{i}^{(h)}=h_{i}^{\dagger} h_{i}$ is the holon number at site $i$, equal to 1 or 0 . This decoupling scheme is called as the $\mathrm{CP}^{1}$ representation ${ }^{30}$. The advantage of this formalism is that the charge and spin degrees of freedom of the electron may be separated at the mean field (MF) level, where the elementary charge and spin excitations are called the holon and spinon, respectively. We call such holon and spinon as the bare holon and spinon, respectively, since an extra $U(1)$ gauge degree of freedom related with the constraint $\sum_{\sigma} a_{i \sigma}^{\dagger} a_{i \sigma}=1$ appears, i.e., the $\mathrm{CP}^{1}$ representation is invariant under a local $U(1)$ gauge transformation,

$$
h_{i} \rightarrow h_{i} e^{i \theta_{i}}, \quad a_{i \sigma} \rightarrow a_{i \sigma} e^{i \theta_{i}},
$$

and then all physical quantities should be invariant with respect to this transformation. Thus both bare holon $h_{i}$ and spinon $a_{i \sigma}$ are not gauge invariant, and they are strongly coupled by these $U(1)$ gauge field fluctuations. In other words, these bare holon and spinon are not real.

However, the constrained $\mathrm{CP}^{1}$ boson $a_{i \sigma}$ can be mapped exactly onto the pseudospin representation defined with an additional phase factor, this is because the empty and doubly occupied spin states have been ruled out due to the constraint $a_{i \uparrow}^{\dagger} a_{i \uparrow}+a_{i \downarrow}^{\dagger} a_{i \downarrow}=1$, and only the spin-up and spin-down singly occupied spin states are allowed, therefore the original four-dimensional representation space is reduced to a $2 \mathrm{D}$ space. Due to the symmetry of the spin-up and spin-down states, $\mid$ occupied $\rangle_{\uparrow}=\left(\begin{array}{l}1 \\ 0\end{array}\right)_{\uparrow}$ and $\mid$ empty $\rangle_{\uparrow}=\left(\begin{array}{l}0 \\ 1\end{array}\right)_{\uparrow}$ are singlyoccupied and empty spin-up, while $\mid$ occupied $\rangle_{\downarrow}=\left(\begin{array}{l}0 \\ 1\end{array}\right)_{\downarrow}$ and $\mid$ empty $\rangle_{\downarrow}=\left(\begin{array}{l}1 \\ 0\end{array}\right)_{\downarrow}$ are singly-occupied and empty spin-down states, respectively, thus the constrained $\mathrm{CP}^{1}$ boson operators $a_{i \sigma}$ can be represented in this reduced $2 \mathrm{D}$ space as,

$$
\begin{aligned}
a_{\uparrow} & \left.=e^{i \Phi_{\uparrow}} \mid \text { occupied }\right\rangle_{\downarrow}\langle\text { occupied }| \\
& =e^{i \Phi_{\uparrow}}\left(\begin{array}{ll}
0 & 0 \\
1 & 0
\end{array}\right)=e^{i \Phi_{\uparrow}} S^{-},
\end{aligned}
$$




$$
\begin{aligned}
a_{\downarrow} & \left.=e^{i \Phi_{\downarrow}} \mid \text { occupied }\right\rangle_{\uparrow} \downarrow\langle\text { occupied }| \\
& =e^{i \Phi_{\downarrow}}\left(\begin{array}{ll}
0 & 1 \\
0 & 0
\end{array}\right)=e^{i \Phi_{\downarrow}} S^{+},
\end{aligned}
$$

where $S^{-}$is the $S^{z}$ lowering operator, while $S^{+}$is the $S^{z}$ raising operator, then the constraint $\sum_{\sigma} a_{i \sigma}^{\dagger} a_{i \sigma}=$ $S_{i}^{+} S_{i}^{-}+S_{i}^{-} S_{i}^{+}=1$ is exactly satisfied. Obviously, the bare spinon contains both phase and amplitude parts, and the phase part is described by the phase factor $e^{i \Phi_{i \sigma}}$, while the amplitude part is described by the spin operator $S_{i}$. In this case, the electron decoupling form (2) with the constraint can be expressed as,

$$
C_{i \uparrow}=h_{i}^{\dagger} e^{i \Phi_{i \uparrow}} S_{i}^{-}, \quad C_{i \downarrow}=h_{i}^{\dagger} e^{i \Phi_{i \downarrow}} S_{i}^{+},
$$

with the local $U(1)$ gauge transformation (4) is rewritten as,

$$
h_{i} \rightarrow h_{i} e^{i \theta_{i}}, \quad \Phi_{i \sigma} \rightarrow \Phi_{i \sigma}+\theta_{i}
$$

Moreover, the phase factor of the bare spinon $e^{i \Phi_{i \sigma}}$ can be incorporated into the bare holon, thus we obtain a new fermion-spin transformation from Eq. (6) as,

$$
C_{i \uparrow}=h_{i \uparrow}^{\dagger} S_{i}^{-}, \quad C_{i \downarrow}=h_{i \downarrow}^{\dagger} S_{i}^{+},
$$

with the spinful fermion operator $h_{i \sigma}=e^{-i \Phi_{i \sigma}} h_{i}$ describes the charge degree of freedom together with the phase part of the spin degree of freedom (dressed holon), while the spin operator $S_{i}$ describes the amplitude part of the spin degree of freedom (dressed spinon). This electron decoupling form (8) is called as a partial CSS since only the amplitude part of the spin degree of freedom is separated from the electron operator. These dressed holon and spinon are invariant under the local $U(1)$ gauge transformation (7), and therefore all physical quantities from the dressed holon and spinon also are invariant with respect to this gauge transformation. In this sense, the dressed holon and spinon are real. The dressed holon carries a spinon cloud (magnetic flux), and is a magnetic dressing ${ }^{24}$. In other words, the dressed holon carries some spinon messages, i.e., it shares some effects of spinon configuration rearrangements due to the presence of the hole itself. We emphasize that the dressed holon $h_{i \sigma}=e^{-i \Phi_{i \sigma}} h_{i}$ is the spinless fermion $h_{i}$ (bare holon) incorporated in the spinon cloud $e^{-i \Phi_{i \sigma}}$ (magnetic flux). Although in the common sense $h_{i \sigma}$ is not an real spinful fermion operator, it behaves like a spinful fermion. In correspondence with these special physical properties, we find that $h_{i \sigma}^{\dagger} h_{i \sigma}=h_{i}^{\dagger} e^{i \Phi_{i \sigma}} e^{-i \Phi_{i \sigma}} h_{i}=h_{i}^{\dagger} h_{i}$, which guarantees that the electron constraint, $\sum_{\sigma} C_{i \sigma}^{\dagger} C_{i \sigma}=$ $S_{i}^{+} h_{i \uparrow} h_{i \uparrow}^{\dagger} S_{i}^{-}+S_{i}^{-} h_{i \downarrow} h_{i \downarrow}^{\dagger} S_{i}^{+}=h_{i} h_{i}^{\dagger}\left(S_{i}^{+} S_{i}^{-}+S_{i}^{-} S_{i}^{+}\right)=$ $1-h_{i}^{\dagger} h_{i} \leq 1$, is always satisfied in analytical calculations. Moreover the double spinful fermion occupancy, $h_{i \sigma}^{\dagger} h_{i-\sigma}^{\dagger}=e^{i \Phi_{i \sigma}} h_{i}^{\dagger} h_{i}^{\dagger} e^{i \Phi_{i-\sigma}}=0$ and $h_{i \sigma} h_{i-\sigma}=$ $e^{-i \Phi_{i \sigma}} h_{i} h_{i} e^{-i \Phi_{i-\sigma}}=0$, is ruled out automatically. Since the spinless fermion $h_{i}$ and spin operators $S_{i}^{+}$and $S_{i}^{-}$ obey the anticommutation relation and Pauli spin algebra, respectively, it is then easy to show that the spinful fermion $h_{i \sigma}$ also obeys the same anticommutation relation as the spinless fermion $h_{i}$.

Although the choice of the $\mathrm{CP}^{1}$ representation is convenient, so long as $h_{i}^{\dagger} h_{i}=1, \sum_{\sigma} C_{i \sigma}^{\dagger} C_{i \sigma}=0$, no matter what the values of $S_{i}^{+} S_{i}^{-}$and $S_{i}^{-} S_{i}^{+}$are, therefore a "spin" even to an empty site has been assigned. It has been shown ${ }^{29}$ that this defect can be cured by introducing a projection operator $P_{i}$, i.e., the constrained electron operator can be mapped exactly using the fermionspin transformation (8) defined with an additional projection operator $P_{i}$. However, this projection operator is cumbersome to handle in the many cases, and it has been dropped in the actual calculations ${ }^{29,31}$. It has been shown $29,31,32$ that such treatment leads to errors of the order $x$ in counting the number of spin states, which is negligible for small dopings. Moreover, the electron constraint is still exactly obeyed even in the MF approximation (MFA), and therefore the essential physics of the gauge invariant dressed holon and spinon is kept. This is because the constrained electron operator $C_{i \sigma}$ in the $t$ - $J$ type model can also be mapped onto the slavefermion formalism ${ }^{31}$ as $C_{i \sigma}=h_{i}^{\dagger} b_{i \sigma}$ with the constraint $h_{i}^{\dagger} h_{i}+\sum_{\sigma} b_{i \sigma}^{\dagger} b_{i \sigma}=1$. We can solve this constraint by rewriting the boson operators $b_{i \sigma}$ in terms of the $\mathrm{CP}^{1}$ boson operators $a_{i \sigma}$ as $b_{i \sigma}=a_{i \sigma} \sqrt{1-h_{i}^{\dagger} h_{i}}$ supplemented by the constraint $\sum_{\sigma} a_{i \sigma}^{\dagger} a_{i \sigma}=1$. As mentioned above, the $\mathrm{CP}^{1}$ boson operators $a_{i \uparrow}$ and $a_{i \downarrow}$ with the constraint can be identified with the pseudospin lowering and raising operators, respectively, defined with the additional phase factor, therefore the projection operator is approximately related to the holon number operator by $P_{i} \sim \sqrt{1-h_{i \sigma}^{\dagger} h_{i \sigma}}=\sqrt{1-h_{i}^{\dagger} h_{i}}$, and its main role is to remove the spurious spin when there is a holon at the site $i$.

\section{DRESSED HOLON AND SPINON GREEN'S FUNCTIONS}

Before discussing the charge and spin dynamics, let us first calculate the dressed holon and spinon Green's functions. The low-energy behavior of the $t-t^{\prime}-J$ model in the partial CSS fermion-spin representation can be expressed as ${ }^{29,31}$,

$$
\begin{aligned}
H= & -t \sum_{i \hat{\eta}}\left(h_{i \uparrow} S_{i}^{+} h_{i+\hat{\eta} \uparrow}^{\dagger} S_{i+\hat{\eta}}^{-}+h_{i \downarrow} S_{i}^{-} h_{i+\hat{\eta} \downarrow}^{\dagger} S_{i+\hat{\eta}}^{+}\right) \\
& +t^{\prime} \sum_{i \hat{\tau}}\left(h_{i \uparrow} S_{i}^{+} h_{i+\hat{\tau} \uparrow}^{\dagger} S_{i+\hat{\tau}}^{-}+h_{i \downarrow} S_{i}^{-} h_{i+\hat{\tau} \downarrow}^{\dagger} S_{i+\hat{\tau}}^{+}\right) \\
& -\mu \sum_{i \sigma} h_{i \sigma}^{\dagger} h_{i \sigma}+J_{\mathrm{eff}} \sum_{i \hat{\eta}} \mathbf{S}_{i} \cdot \mathbf{S}_{i+\hat{\eta}} .
\end{aligned}
$$

where $J_{\text {eff }}=(1-x)^{2} J$, and $x=\left\langle h_{i \sigma}^{\dagger} h_{i \sigma}\right\rangle=\left\langle h_{i}^{\dagger} h_{i}\right\rangle$ is the hole doping concentration. As a consequence, the kinetic 
part in the $t-t^{\prime}-J$ model has been expressed as the dressed holon-spinon interaction, which dominates the essential physics of the underdoped cuprates. The one-particle dressed holon and spinon two-time Green's functions are defined as,

$$
\begin{aligned}
g_{\sigma}\left(i-j, t-t^{\prime}\right) & =-i \theta\left(t-t^{\prime}\right)\left\langle\left[h_{i \sigma}(t), h_{j \sigma}^{\dagger}\left(t^{\prime}\right)\right]\right\rangle \\
& =\left\langle\left\langle h_{i \sigma}(t) ; h_{j \sigma}^{\dagger}\left(t^{\prime}\right)\right\rangle\right\rangle, \\
D\left(i-j, t-t^{\prime}\right) & =-i \theta\left(t-t^{\prime}\right)\left\langle\left[S_{i}^{+}(t), S_{j}^{-}\left(t^{\prime}\right)\right]\right\rangle \\
& =\left\langle\left\langle S_{i}^{+}(t) ; S_{j}^{-}\left(t^{\prime}\right)\right\rangle\right\rangle,
\end{aligned}
$$

respectively, where $\langle\ldots\rangle$ is an average over the ensemble.

\section{A. Equation of motion}

Since the dressed spinon operators obey Pauli algebra, our goal is to evaluate the dressed holon and spinon Green's functions directly for the fermion and spin operators in terms of the equation of motion method. In the framework of the equation of motion, the time-Fourier transform of the two-time Green's function $G(\omega)=\left\langle\left\langle A ; A^{\dagger}\right\rangle\right\rangle_{\omega}$ satisfies the equation ${ }^{33}$, $\omega\left\langle\left\langle A ; A^{\dagger}\right\rangle\right\rangle_{\omega}=\left\langle\left[A, A^{\dagger}\right]\right\rangle+\left\langle\left\langle[A, H] ; A^{\dagger}\right\rangle\right\rangle_{\omega}$. If we define the orthogonal operator $L$ as, $[A, H]=\zeta A-i L$ with $\left\langle\left[L, A^{\dagger}\right]\right\rangle=0$, the full Green's function can be expressed as,

$$
G(\omega)=G^{(0)}(\omega)+\frac{1}{\varsigma^{2}} G^{(0)}(\omega)\left\langle\left\langle L ; L^{\dagger}\right\rangle\right\rangle_{\omega} G^{(0)}(\omega),
$$

where $\varsigma=\left\langle\left[A, A^{\dagger}\right]\right\rangle$, and the MF Green's function $G^{(0)-1}(\omega)=(\omega-\zeta) / \varsigma$. It has been shown ${ }^{33}$ that if the self-energy $\Sigma(\omega)$ is identified as the irreducible part of $\left\langle\left\langle L ; L^{\dagger}\right\rangle\right\rangle_{\omega}$, the full Green's function (11) can be evaluated as,

$$
G(\omega)=\frac{\varsigma}{\omega-\zeta-\Sigma(\omega)},
$$

with $\Sigma(\omega)=\left\langle\left\langle L ; L^{\dagger}\right\rangle\right\rangle_{\omega}^{i r r} / \varsigma$. In the framework of the diagrammatic technique, $\Sigma(\omega)$ corresponds to the contribution of irreducible diagrams.

\section{B. The mean-field theory}

Within MFA, the $t-t^{\prime}-J$ model (9) can be decoupled as,

$$
\begin{aligned}
H_{M F A} & =H_{t}+H_{J}-8 N t \chi_{1} \phi_{1}+8 N t^{\prime} \chi_{2} \phi_{2}, \\
H_{t} & =\chi_{1} t \sum_{i \hat{\eta} \sigma} h_{i+\hat{\eta} \sigma}^{\dagger} h_{i \sigma}-\chi_{2} t^{\prime} \sum_{i \hat{\tau} \sigma} h_{i+\hat{\tau} \sigma}^{\dagger} h_{i \sigma} \\
& -\mu \sum_{i \sigma} h_{i \sigma}^{\dagger} h_{i \sigma}, \\
H_{J} & =J_{\mathrm{eff}} \sum_{i \hat{\eta}}\left[\frac{1}{2} \epsilon\left(S_{i}^{+} S_{i+\hat{\eta}}^{-}+S_{i}^{-} S_{i+\hat{\eta}}^{+}\right)+S_{i}^{Z} S_{i+\hat{\eta}}^{Z}\right]
\end{aligned}
$$

$$
-\phi_{2} t^{\prime} \sum_{i \hat{\tau}}\left(S_{i}^{+} S_{i+\hat{\tau}}^{-}+S_{i}^{-} S_{i+\hat{\tau}}^{+}\right),
$$

where the dressed holon's particle-hole parameters $\phi_{1}=$ $\left\langle h_{i \sigma}^{\dagger} h_{i+\hat{\eta} \sigma}\right\rangle$ and $\phi_{2}=\left\langle h_{i \sigma}^{\dagger} h_{i+\hat{\tau} \sigma}\right\rangle$, the dressed spinon correlation functions $\chi_{1}=\left\langle S_{i}^{+} S_{i+\hat{\eta}}^{-}\right\rangle$and $\chi_{2}=\left\langle S_{i}^{+} S_{i+\hat{\tau}}^{-}\right\rangle$, and $\epsilon=1+2 t \phi_{1} / J_{\text {eff }}$. Since AFLRO in the undoped cuprates is destroyed $^{34}$ by hole doping of the order $\sim$ 0.024, there is therefore no AFLRO in the doped regime $x \geq 0.025$, i.e., $\left\langle S_{i}^{z}\right\rangle=0$, and a disordered spin liquid state emerges. It has been argued that this spin liquid state may play a crucial role in the mechanism for $\mathrm{HTSC}^{3,4}$. In this paper, we focus on the normal-state properties in the doped regime without AFLRO. In this case, a similar MF theory ${ }^{35}$ of the $t-J$ model based on the fermion-spin theory has been discussed within the Kondo-Yamaji decoupling scheme ${ }^{36}$, which is a stage onestep further than the Tyablikov's decoupling scheme ${ }^{37}$. In this MF theory ${ }^{35}$, the phase factor $e^{i \Phi_{i \sigma}}$ describing the phase part of the spin degree of freedom was not considered. Following their discussions ${ }^{35}$, we obtain the MF dressed holon and spinon Green's functions in the present case as,

$$
\begin{aligned}
g_{\sigma}^{(0)}(\mathbf{k}, \omega) & =\frac{1}{\omega-\xi_{k}}, \\
D^{(0)}(\mathbf{k}, \omega) & =\frac{B_{k}}{\omega^{2}-\omega_{k}^{2}},
\end{aligned}
$$

respectively, where $B_{k}=\lambda_{1}\left[2 \chi_{1}^{z}\left(\epsilon \gamma_{\mathbf{k}}-1\right)+\chi_{1}\left(\gamma_{\mathbf{k}}-\right.\right.$ $\epsilon)]-\lambda_{2}\left(2 \chi_{2}^{z} \gamma_{\mathbf{k}}^{\prime}-\chi_{2}\right), \lambda_{1}=2 Z J_{e f f}, \lambda_{2}=4 Z \phi_{2} t^{\prime}$, $\gamma_{\mathbf{k}}=(1 / Z) \sum_{\hat{\eta}} e^{i \mathbf{k} \cdot \hat{\eta}}, \gamma_{\mathbf{k}}^{\prime}=(1 / Z) \sum_{\hat{\tau}} e^{i \mathbf{k} \cdot \hat{\tau}}, Z$ is the number of the nearest neighbor or second-nearest neighbor sites, and the MF dressed holon and spinon excitation spectra are given by,

$$
\begin{aligned}
\xi_{k} & =Z t \chi_{1} \gamma_{\mathbf{k}}-Z t^{\prime} \chi_{2} \gamma_{\mathbf{k}}^{\prime}-\mu, \\
\omega_{k}^{2} & =A_{1}\left(\gamma_{k}\right)^{2}+A_{2}\left(\gamma_{k}^{\prime}\right)^{2}+A_{3} \gamma_{k} \gamma_{k}^{\prime} \\
& +A_{4} \gamma_{k}+A_{5} \gamma_{k}^{\prime}+A_{6},
\end{aligned}
$$

respectively, with $A_{1}=\alpha \epsilon \lambda_{1}^{2}\left(\epsilon \chi_{1}^{z}+\chi_{1} / 2\right), A_{2}=$ $\alpha \lambda_{2}^{2} \chi_{2}^{z}, \quad A_{3}=-\alpha \lambda_{1} \lambda_{2}\left(\epsilon \chi_{1}^{z}+\epsilon \chi_{2}^{z}+\chi_{1} / 2\right), \quad A_{4}=$ $-\epsilon \lambda_{1}^{2}\left[\alpha\left(\chi_{1}^{z}+\epsilon \chi_{1} / 2\right)+\left(\alpha C_{1}^{z}+(1-\alpha) /(4 Z)-\alpha \epsilon \chi_{1} /(2 Z)\right)+\right.$ $\left.\left(\alpha C_{1}+(1-\alpha) /(2 Z)-\alpha \chi_{1}^{z} / 2\right) / 2\right]+\alpha \lambda_{1} \lambda_{2}\left(C_{3}+\right.$ $\left.\epsilon \chi_{2}\right) / 2, \quad A_{5}=-3 \alpha \lambda_{2}^{2} \chi_{2} /(2 Z)+\alpha \lambda_{1} \lambda_{2}\left(\chi_{1}^{z}+\epsilon \chi_{1} / 2+\right.$ $\left.C_{3}^{z}\right), \quad A_{6}=\lambda_{1}^{2}\left[\alpha C_{1}^{z}+(1-\alpha) /(4 Z)-\alpha \epsilon \chi_{1} /(2 Z)+\right.$ $\left.\epsilon^{2}\left(\alpha C_{1}+(1-\alpha) /(2 Z)-\alpha \chi_{1}^{z} / 2\right) / 2\right]+\lambda_{2}^{2}\left(\alpha C_{2}+\right.$ $\left.\left.(1-\alpha) /(2 Z)-\alpha \chi_{2}^{z} / 2\right) / 2\right)-\alpha \epsilon \lambda_{1} \lambda_{2} C_{3}$, and the spinon correlation functions $\chi_{1}^{z}=\left\langle S_{i}^{z} S_{i+\hat{\eta}}^{z}\right\rangle, \chi_{2}^{z}=$ $\left\langle S_{i}^{z} S_{i+\hat{\tau}}^{z}\right\rangle, \quad C_{1}=\left(1 / Z^{2}\right) \sum_{\hat{\eta}, \hat{\eta}^{\prime}}\left\langle S_{i+\hat{\eta}}^{+} S_{i+\hat{\eta}^{\prime}}^{-}\right\rangle, \quad C_{1}^{z}=$ $\left(1 / Z^{2}\right) \sum_{\hat{\eta}, \hat{\eta}^{\prime}}\left\langle S_{i+\hat{\eta}}^{z} S_{i+\hat{\eta}^{\prime}}^{z}\right\rangle, C_{2}=\left(1 / Z^{2}\right) \sum_{\hat{\tau}, \hat{\tau^{\prime}}}\left\langle S_{i+\hat{\tau}^{\prime}}^{+} S_{i+\hat{\tau}^{\prime}}^{-}\right\rangle$, $C_{3} \quad=\quad(1 / Z) \sum_{\hat{\tau}}\left\langle S_{i+\hat{\eta}}^{+} S_{i+\hat{\tau}}^{-}\right\rangle, \quad$ and $C_{3}^{z}=(1 / Z) \sum_{\hat{\tau}}\left\langle S_{i+\hat{\eta}}^{z} S_{i+\hat{\tau}}^{z}\right\rangle$. In order not to violate the sum rule of the correlation function $\left\langle S_{i}^{+} S_{i}^{-}\right\rangle=1 / 2$ in the case without AFLRO, the important decoupling parameter $\alpha$ has been introduced in the MF calculation ${ }^{35,36}$, which can be regarded as the vertex correction. All 
the above MF order parameters, decoupling parameter $\alpha$, and chemical potential $\mu$ are determined by the selfconsistent calculation ${ }^{35}$.

\section{The dressed holon and spinon self-energies}

With the help of Eq. (12), the full dressed holon and spinon Green's functions of the $t-t^{\prime}-J$ model (9) are expressed as,

$$
\begin{aligned}
g_{\sigma}(\mathbf{k}, \omega) & =\frac{1}{\omega-\xi_{k}-\Sigma_{h}^{(2)}(\mathbf{k}, \omega)} \\
D(\mathbf{k}, \omega) & =\frac{B_{k}}{\omega^{2}-\omega_{k}^{2}-\Sigma_{s}^{(2)}(\mathbf{k}, \omega)}
\end{aligned}
$$

respectively, where the second-order dressed holon self-energy from the dressed spinon pair bubble $\Sigma_{h}^{(2)}(\mathbf{k}, \omega)=\left\langle\left\langle L_{k}^{(h)}(t) ; L_{k}^{(h) \dagger}\left(t^{\prime}\right)\right\rangle\right\rangle_{\omega}$ with the orthogonal operator $L_{i}^{(h)}=-t \sum_{\hat{\eta}} h_{i+\hat{\eta} \sigma}\left(S_{i+\hat{\eta}}^{-} S_{i}^{+}-\chi_{1}\right)+$ $t^{\prime} \sum_{\hat{\tau}} h_{i+\hat{\tau} \sigma}\left(S_{i+\hat{\tau}}^{-} S_{i}^{+}-\chi_{2}\right)$, and can be evaluated $\mathrm{as}^{31}$,

$$
\begin{aligned}
\Sigma_{h}^{(2)}(\mathbf{k}, \omega) & =\frac{1}{2}\left(\frac{Z}{N}\right)^{2} \sum_{p p^{\prime}} \gamma_{12}^{2}\left(\mathbf{k}, \mathbf{p}, \mathbf{p}^{\prime}\right) \frac{B_{p^{\prime}} B_{p+p^{\prime}}}{4 \omega_{p^{\prime}} \omega_{p+p^{\prime}}} \\
& \times\left(\frac{F_{1}^{(h)}\left(k, p, p^{\prime}\right)}{\omega+\omega_{p+p^{\prime}}-\omega_{p^{\prime}}-\xi_{p+k}}\right. \\
& +\frac{F_{2}^{(h)}\left(k, p, p^{\prime}\right)}{\omega+\omega_{p^{\prime}}-\omega_{p+p^{\prime}}-\xi_{p+k}} \\
& +\frac{F_{3}^{(h)}\left(k, p, p^{\prime}\right)}{\omega+\omega_{p^{\prime}}+\omega_{p+p^{\prime}}-\xi_{p+k}} \\
& \left.-\frac{F_{4}^{(h)}\left(k, p, p^{\prime}\right)}{\omega-\omega_{p+p^{\prime}}-\omega_{p^{\prime}}-\xi_{p+k}}\right)
\end{aligned}
$$

where $\gamma_{12}^{2}\left(\mathbf{k}, \mathbf{p}, \mathbf{p}^{\prime}\right)=\left[\left(t \gamma_{\mathbf{p}^{\prime}+\mathbf{p}+\mathbf{k}}-t^{\prime} \gamma_{\mathbf{p}^{\prime}+\mathbf{p}+\mathbf{k}}^{\prime}\right)^{2}+\right.$ $\left.\left(t \gamma_{\mathbf{p}^{\prime}-\mathbf{k}}-t^{\prime} \gamma_{\mathbf{p}^{\prime}-\mathbf{k}}^{\prime}\right)^{2}\right], F_{1}^{(h)}\left(k, p, p^{\prime}\right)=n_{F}\left(\xi_{p+k}\right)\left[n_{B}\left(\omega_{p^{\prime}}\right)-\right.$ $\left.n_{B}\left(\omega_{p+p^{\prime}}\right)\right]+n_{B}\left(\omega_{p+p^{\prime}}\right)\left[1+n_{B}\left(\omega_{p^{\prime}}\right)\right], \quad F_{2}^{(h)}\left(k, p, p^{\prime}\right)=$ $n_{F}\left(\xi_{p+k}\right)\left[n_{B}\left(\omega_{p^{\prime}+p}\right)-n_{B}\left(\omega_{p^{\prime}}\right)\right]+n_{B}\left(\omega_{p^{\prime}}\right)\left[1+n_{B}\left(\omega_{p^{\prime}+p}\right)\right]$, $F_{3}^{(h)}\left(k, p, p^{\prime}\right)=n_{F}\left(\xi_{p+k}\right)\left[1+n_{B}\left(\omega_{p+p^{\prime}}\right)+n_{B}\left(\omega_{p^{\prime}}\right)\right]+$ $n_{B}\left(\omega_{p^{\prime}}\right) n_{B}\left(\omega_{p+p^{\prime}}\right), \quad F_{4}^{(h)}\left(k, p, p^{\prime}\right)=n_{F}\left(\xi_{p+k)}[1+\right.$ $\left.n_{B}\left(\omega_{p+p^{\prime}}\right)+n_{B}\left(\omega_{p^{\prime}}\right)\right]-\left[1+n_{B}\left(\omega_{p^{\prime}}\right)\right]\left[1+n_{B}\left(\omega_{p+p^{\prime}}\right)\right]$, and $n_{B}\left(\omega_{p}\right)$ and $n_{F}\left(\xi_{p}\right)$ are the boson and fermion distribution functions, respectively. This dressed holon selfenergy is ascribed purely to the dressed holon-spinon interaction, and characterizes the competition between the kinetic energy and magnetic energy. The calculation of the dressed spinon self-energy is quite tedious ${ }^{38}$, since our starting point is the dressed spinon MF solution ${ }^{35}$ within the Kondo-Yamaji decoupling scheme in subsection B. From Eq. (11) and the MF dressed spinon Green's function (14b), the full dressed spinon Green's function satisfies the relation ${ }^{38}, \omega^{2} D(k, \omega)=B_{k}+$ $\left\langle\left\langle\left[\left[S_{i}^{+}(t), H(t)\right], H(t)\right] ; S_{j}^{-}\left(t^{\prime}\right)\right\rangle\right\rangle_{k, \omega}$ with $\left[\left[S_{i}^{+}, H\right], H\right]_{k}=$ $\omega_{k}^{2} S_{k}^{+}-i \Gamma_{k}^{(s)}$. In the disordered liquid state without AFLRO, the dressed holon-spinon interaction should dominate the essential physics ${ }^{38}$. In this case, the orthogonal operator $L_{k}^{(s)}$ for the dressed spinon can be selected from $\Gamma_{k}^{(s)}$ as $^{38}$,

$$
\begin{aligned}
L_{i}^{(s)} & =-\left(2 \epsilon \chi_{1}^{z}+\chi_{1}\right) \lambda_{1} \frac{1}{Z} \sum_{\hat{\eta}, \hat{a}} t_{\hat{a}}\left(h_{i+\hat{\eta} \uparrow}^{\dagger} h_{i+\hat{\eta}+\hat{a} \uparrow}\right. \\
& \left.+h_{i+\hat{\eta}+\hat{a} \downarrow}^{\dagger} h_{i+\hat{\eta} \downarrow}-2 \phi_{\hat{a}}\right) S_{i+\hat{\eta}+\hat{a}}^{+} \\
& +\left[\left(2 \chi_{1}^{z}+\epsilon \chi_{1}\right) \lambda_{1}-\chi_{2} \lambda_{2}\right] \sum_{\hat{a}} t_{\hat{a}}\left(h_{i \uparrow}^{\dagger} h_{i+\hat{a} \uparrow}\right. \\
& \left.+h_{i+\hat{a} \downarrow}^{\dagger} h_{i \downarrow}-2 \phi_{\hat{a}}\right) S_{i+\hat{a}}^{+} \\
& +2 \chi_{2}^{z} \lambda_{2} \frac{1}{Z} \sum_{\hat{\tau}, \hat{a}} t_{\hat{a}}\left(h_{i+\hat{\tau} \uparrow}^{\dagger} h_{i+\hat{\tau}+\hat{a} \uparrow}\right. \\
& \left.+h_{i+\hat{\tau}+\hat{a} \downarrow}^{\dagger} h_{i+\hat{\tau} \downarrow}-2 \phi_{\hat{a}}\right) S_{i+\hat{\tau}+\hat{a}}^{+},
\end{aligned}
$$

where $\hat{a}=\hat{\eta}, \hat{\tau}$, with $t_{\hat{\eta}}=t, \phi_{\hat{\eta}}=\phi_{1}$, and $t_{\hat{\tau}}=-t^{\prime}$, $\phi_{\hat{\tau}}=\phi_{2}$. Following [38], we obtain the dressed spinon self-energy $\Sigma_{s}^{(2)}(\mathbf{k}, \omega)=\left\langle\left\langle L_{i}^{(s)}(t) ; L_{j}^{(s) \dagger}\left(t^{\prime}\right)\right\rangle\right\rangle_{k, \omega}$,

$$
\begin{aligned}
\Sigma_{s}^{(2)}(\mathbf{k}, \omega) & =B_{k}\left(\frac{Z}{N}\right)^{2} \sum_{p p^{\prime}} \gamma_{12}^{2}\left(\mathbf{k}, \mathbf{p}, \mathbf{p}^{\prime}\right) \frac{B_{k+p}}{2 \omega_{k+p}} \\
& \times\left(\frac{F_{1}^{(s)}\left(k, p, p^{\prime}\right)}{\omega+\xi_{p+p^{\prime}}-\xi_{p^{\prime}}-\omega_{k+p}}\right. \\
& \left.-\frac{F_{2}^{(s)}\left(k, p, p^{\prime}\right)}{\omega+\xi_{p+p^{\prime}}-\xi_{p^{\prime}}+\omega_{k+p}}\right)
\end{aligned}
$$

with $\quad F_{1}^{(s)}\left(k, p, p^{\prime}\right)=$ $n_{F}\left(\xi_{p+p^{\prime}}\right)\left[1-n_{F}\left(\xi_{p^{\prime}}\right)\right]-n_{B}\left(\omega_{k+p}\right)\left[n_{F}\left(\xi_{p^{\prime}}\right)-n_{F}\left(\xi_{p+p^{\prime}}\right)\right]$, and $F_{2}^{(s)}\left(k, p, p^{\prime}\right)=n_{F}\left(\xi_{p+p^{\prime}}\right)\left[1-n_{F}\left(\xi_{p^{\prime}}\right)\right]+[1+$ $\left.n_{B}\left(\omega_{k+p}\right)\right]\left[n_{F}\left(\xi_{p^{\prime}}\right)-n_{F}\left(\xi_{p+p^{\prime}}\right)\right]$. Within the diagrammatic technique, this dressed spinon self-energy $\Sigma_{s}^{(2)}(\mathbf{k}, \omega)$ corresponds to the contribution from the dressed holon pair bubble, and is consistent with our previous result ${ }^{38}$.

\section{CHARGE TRANSPORT}

Recently, the emergence and evolution of the metallic transport in doped cuprates have been extensively studied by virtue of systematic transport measurements ${ }^{14}$. It is shown that the resistivity shows a crossover from the low temperature insulating-like to moderate temperature metallic-like behavior in the heavily underdoped regime $(0.025 \leq x<0.055)$, and a temperature linear dependence with deviations at low temperatures in the underdoped regime $(0.055<x<0.15)$. These striking behaviors have been found to be intriguingly related to the $\mathrm{AF}$ correlation $^{14}$. In this case, a natural question 
is what is the physical origin of this transport transformation from the insulating liquid state in the heavily underdoped regime to the unusual metallic state in the underdoped regime? In this section, we try to discuss this issue. Since the local constraint has been treated properly in the partial CSS fermion-spin theory, the extra $U(1)$ gauge degree of freedom related with the local constraint is incorporated into the dressed holon as mentioned in Sec. II. In this case, the external electronic field only is coupled to the dressed holons, and the conductivity is given by,

$$
\sigma(\omega)=-\frac{\operatorname{Im}_{h}(\omega)}{\omega},
$$

with $\Pi_{h}(\omega)$ is the dressed holon current-current correlation function, and is defined as $\Pi_{h}\left(t-t^{\prime}\right)=$ $\left\langle\left\langle j_{h}(t) j_{h}\left(t^{\prime}\right)\right\rangle\right\rangle$, where the current density of the dressed holons is obtained by taking the time derivation of the polarization operator with the use of the equation of motion $\mathrm{as}^{31}, j_{h}=\left(e \chi_{1} t / 2\right) \sum_{i \hat{\eta} \sigma} \hat{\eta} h_{i+\hat{\eta} \sigma}^{\dagger} h_{i \sigma}-$ $\left(e \chi_{2} t^{\prime} / 2\right) \sum_{i \hat{\tau} \sigma} \hat{\tau} h_{i+\hat{\tau} \sigma}^{\dagger} h_{i \sigma}$. With the help of the full dressed holon Green's function (16a), the current-current correlation function is evaluated as,

$$
\begin{aligned}
\Pi_{h}\left(i \omega_{n}\right) & =-\left(\frac{Z e}{2}\right)^{2} \frac{1}{N} \sum_{k} \gamma_{s}^{2}(\mathbf{k}) \frac{1}{\beta} \sum_{i \omega_{m}^{\prime} \sigma} g_{\sigma}\left(\mathbf{k}, i \omega_{m}^{\prime}+i \omega_{n}\right) \\
& \times g_{\sigma}\left(\mathbf{k}, i \omega_{m}^{\prime}\right),
\end{aligned}
$$

where

$i \omega_{n}$ is the Matsubara frequency, $\gamma_{s}^{2}(\mathbf{k})=\left[\sin ^{2} k_{x}\left(\chi_{1} t-\right.\right.$ $\left.\left.2 \chi_{2} t^{\prime} \cos k_{y}\right)^{2}+\sin ^{2} k_{y}\left(\chi_{1} t-2 \chi_{2} t^{\prime} \cos k_{x}\right)^{2}\right] / 4$. The full dressed holon Green's function can be expressed as frequency integrals in terms of the spectral representation,

$$
g_{\sigma}\left(\mathbf{k}, i \omega_{n}\right)=\int_{-\infty}^{\infty} \frac{d \omega}{2 \pi} \frac{A_{\sigma}^{(h)}(\mathbf{k}, \omega)}{i \omega_{n}-\omega}
$$

with the dressed holon spectral function $A_{\sigma}^{(h)}(\mathbf{k}, \omega)=$ $-2 \operatorname{Im} g_{\sigma}(\mathbf{k}, \omega)$. Substituting the Eqs. (22) and (21) into Eq. (20), and evaluating the frequency summation, we obtain the conductivity $\mathrm{as}^{31}$,

$$
\begin{aligned}
\sigma(\omega) & =\left(\frac{Z e}{2}\right)^{2} \frac{1}{N} \sum_{k \sigma} \gamma_{s}^{2}(\mathbf{k}) \int_{-\infty}^{\infty} \frac{d \omega^{\prime}}{2 \pi} A_{\sigma}^{(h)}\left(\mathbf{k}, \omega^{\prime}+\omega\right) \\
& \times A_{\sigma}^{(h)}\left(\mathbf{k}, \omega^{\prime}\right) \frac{n_{F}\left(\omega^{\prime}+\omega\right)-n_{F}\left(\omega^{\prime}\right)}{\omega} .
\end{aligned}
$$

We have performed a numerical calculation for $\sigma(\omega)$, and the results at $x=0.03$ (solid line), $x=0.05$ (dashed line), and $x=0.07$ (dotted line) for $t / J=2.5$ and $t^{\prime} / t=0.15$ with $T=0$ are plotted in Fig. 1, hereinafter the charge $e$ is set as the unit. For a comparison, the experimental result ${ }^{11}$ of $\mathrm{YBa}_{2} \mathrm{Cu}_{3} \mathrm{O}_{7-x}$ is also plotted in Fig. 1 (inset). Our results show that there is a low-energy peak at $\omega<0.3 t$ separated by a gap or pseudogap at $0.3 t$ from a midinfrared band. This midinfrared band is doping dependent, the component increases with increasing dopings for $0.3 t<\omega<1.0 t$ and is nearly independent of dopings for $\omega>1.0 t$, however, the position of the midinfrared peak is shifted to lower energies with increased dopings. This reflects an increase in the mobile carrier density, and indicates that the spectral weight of the midinfrared sideband is taken from the Drude absorption, then the spectral weight from both low energy peak and midinfrared band represent the actual free-carrier density. For a better understanding of the optical properties, we have made a series of calculations for $\sigma(\omega)$ at different temperatures, and the results at $x=0.06$ with $T=0$ (solid line), $T=0.1 J$ (dashed line), and $T=0.3 J$ (dotted line) for $t / J=2.5$ and $t^{\prime} / t=0.15$ are plotted in Fig. 2 in comparison with the experimental data ${ }^{11}$ taken from $\mathrm{YBa}_{2} \mathrm{Cu}_{3} \mathrm{O}_{7-x}$ (inset). It is shown that $\sigma(\omega)$ is temperature-dependent for $\omega<1.0 t$, and almost temperature-independent for $\omega>1.0 t$. The peak at low energies broadens and decreases in the height with increasing temperatures, while the component in the low energy region increases with increasing temperatures, then there is a tendency towards the Drude-like behavior. The midinfrared band is severely suppressed with increasing temperatures, and vanishes at high temperatures, in qualitative agreement with experiments ${ }^{11,12}$.

Now we turn to discuss the resistivity, which is eval-

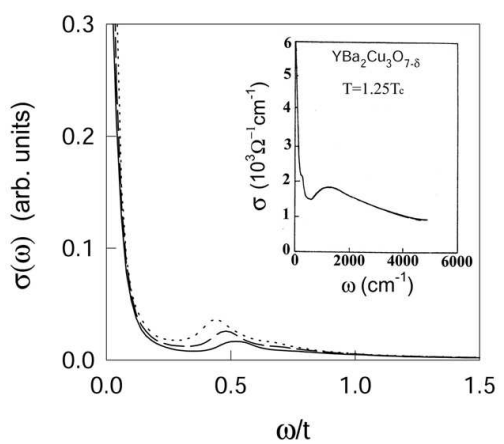

FIG. 1. The conductivity at $x=0.03$ (solid line), $x=0.05$ (dashed line), and $x=0.07$ (dotted line) with $t / J=2.5$ and $t^{\prime} / t=0.15$ in $T=0$. Inset: the experimental result of $\mathrm{YBa}_{2} \mathrm{Cu}_{3} \mathrm{O}_{7-x}$ taken from Ref. [11].

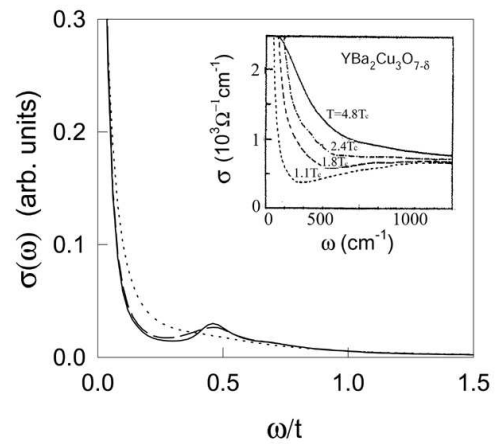

FIG. 2. The conductivity at $x=0.06$ in $T=0$ (solid line), $T=0.1 J$ (dashed line), and $T=0.3 J$ (dotted line) with $t / J=2.5$ and $t^{\prime} / t=0.15$. Inset: the experimental result of $\mathrm{YBa}_{2} \mathrm{Cu}_{3} \mathrm{O}_{7-x}$ taken from Ref. [11]. 
uated as, $\rho=1 / \sigma_{d c}$, with the $d c$ conductivity $\sigma_{d c}$ is obtained from Eq. (23) as $\sigma_{d c}=\lim _{\omega \rightarrow 0} \sigma(\omega)$. This resistivity has been evaluated numerically, and the results are plotted in Fig. 3 as a function of temperature at $x=0.03$ (solid line), $x=0.04$ (dashed line), $x=0.05$ (dotted line), and $x=0.06$ (dash-dotted line) for $t / J=2.5$ and $t^{\prime} / t=0.15$ in comparison with the experimental data ${ }^{14}$ taken from $\mathrm{La}_{2-x} \mathrm{Sr}_{x} \mathrm{CuO}_{4}$ (inset). Our results show obviously that the resistivity is characterized by a crossover from the moderate temperature metallic-like to low temperature insulating-like behavior in the heavily underdoped regime, and a temperature linear dependence with deviations at low temperatures in the underdoped regime. But even in the heavily underdoped regime, the resistivity exhibits the metallic-like behavior over a wide range of temperatures, which also is in qualitative agreement with experiments ${ }^{14}$.

The perovskite parent compound of doped cuprates is a Mott insulator, when holes are doped into this insulator, there is a gain in the kinetic energy per hole proportional to $t$ due to hopping, but at the same time, the spin correlation is destroyed, costing an energy of approximately $J$ per site. Thus doped holes in a Mott insulator can be considered as a competition between the kinetic energy $(x t)$ and magnetic energy $(J)$. The magnetic energy $J$ favors the magnetic order for spins and results in frustration of the kinetic energy, while the kinetic energy $x t$ favors delocalization of holes and tends to destroy the magnetic order. In the present partial CSS fermion-spin theory, the scattering of dressed holons dominates the charge transport, since the scattering rate is obtained from the dressed holon self-energy $\Sigma_{h}^{(2)}(\mathbf{k}, \omega)$, while this self-energy is evaluated by considering the dressed holonspinon interaction, and characterizes a competition between the kinetic energy and magnetic energy. In this case, the striking behavior in the resistivity is intriguingly related to this competition. In the heavily underdoped regime, the dressed holon kinetic energy is much smaller than the dressed spinon magnetic energy at lower

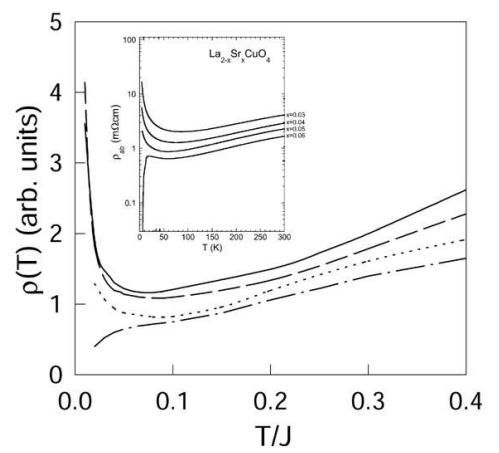

FIG. 3. The electron resistivity as a function of temperature at $x=0.03$ (solid line), $x=0.04$ (dashed line), $x=0.05$ (dotted line), and $x=0.06$ (dash-dotted line) with $t / J=2.5$ and $t^{\prime} / t=0.15$. Inset: the experimental result of $\mathrm{La}_{2-x} \mathrm{Sr}_{x} \mathrm{CuO}_{4}$ taken from Ref. [14]. temperatures due to the strong $\mathrm{AF}$ correlation, where the dressed holons are localized, and the scattering rate from the dressed holon self-energy is severely reduced, this leads to the insulating-like behavior in the resistivity. With increasing temperatures, the dressed holon kinetic energy is increased, while the dressed spinon magnetic energy is decreased. In the region where the dressed holon kinetic energy is larger than the dressed spinon magnetic energy at moderate temperatures, the dressed holons can move in the background of the dressed spinon fluctuation, then the dressed holon scattering would give rise to the metallic-like behavior in the resistivity. Since the charge transport is governed by the dressed holon scattering, then $x$ dressed holons are responsible for the electron conductivity.

\section{INCOMMENSURATE SPIN DYNAMICS}

The interplay between AF correlation and HTSC in doped cuprates is now well-established ${ }^{1}$, but its full understanding is still a challenging issue. Experimentally, NMR, NQR, and $\mu S R$ techniques, particularly inelastic neutron scattering, can provide rather detailed information on the spin dynamics of doped cuprates ${ }^{5-10}$. It has been shown ${ }^{7,8}$ that when AFLRO is suppressed, the IC magnetic correlation develops at a quartet of wave vector $[\pi(1 \pm \delta), \pi]$ and $[\pi,(1 \pm \delta) \pi]$, where the incommensurability $\delta$ increases almost linearly with the hole doping concentration $x$ at lower dopings, and saturates at higher dopings. These exotic features are fully confirmed by the data both on the normal and superconducting states ${ }^{7,8}$. It has been argued that the emergence of the IC magnetic correlation is due to dopings ${ }^{39}$. Although a sharp resonance peak at the commensurate $\mathrm{AF}$ wave vector has been observed in some optimally doped samples, this commensurate scattering is the main new feature that appears in the superconducting phase ${ }^{40,8}$. In this section, we only discuss the IC magnetic correlation in the normal-state. Within the present partial CSS fermion-spin theory, the spin fluctuation couples only to the dressed spinons, then the dynamical spin structure factor (DSSF) is obtained in terms of the full dressed spinon Green's function (16b) $\mathrm{as}^{38}$,

$$
\begin{aligned}
S(\mathbf{k}, \omega) & =-2\left[1+n_{B}(\omega)\right] \operatorname{Im} D(k, \omega) \\
& =-2\left[1+n_{B}(\omega)\right] \\
& \times \frac{B_{k} \operatorname{Im} \Sigma_{s}^{(2)}(\mathbf{k}, \omega)}{\left[\omega^{2}-\omega_{k}^{2}-\operatorname{Re} \Sigma_{s}^{(2)}(\mathbf{k}, \omega)\right]^{2}+\left[\operatorname{Im} \Sigma_{s}^{(2)}(\mathbf{k}, \omega)\right]^{2}},
\end{aligned}
$$

where $\operatorname{Im} \Sigma_{s}^{(2)}(\mathbf{k}, \omega)$ and $\operatorname{Re} \Sigma_{s}^{(2)}(\mathbf{k}, \omega)$ are the corresponding imaginary part and real part of the dressed spinon self-energy function $\Sigma_{s}^{(2)}(\mathbf{k}, \omega)$ in Eq. (19).

At the half-filling, the spin fluctuation scattering remains commensurate at the $\mathrm{AF}$ wave vector $\mathbf{Q}=$ $[1 / 2,1 / 2]$ position (hereafter we use units of $[2 \pi, 2 \pi]$ ), 
which is not presented here for the sake of space. Instead, we plot the DSSF spectrum $S(\mathbf{k}, \omega)$ in the $\left(k_{x}, k_{y}\right)$ plane at $x=0.06$ with $T=0.05 \mathrm{~J}$ and $\omega=0.05 \mathrm{~J}$ for $t / J=2.5$ and $t^{\prime} / t=0.15$ in Fig. 4. This result shows that with dopings, there is a commensurate-IC transition in the spin fluctuation geometry, where all IC peaks lie on a circle of radius of $\delta$. Although some IC satellite diagonal peaks appear, the main weight of the IC peaks is in the parallel direction, and these parallel peaks are located at $[(1 \pm \delta) / 2,1 / 2]$ and $[1 / 2,(1 \pm \delta) / 2]$. The IC peaks are very sharp at low temperatures and energies, which means that these low energy excitations have a dynamical coherence length at low temperatures that is larger than the instantaneous correlation length. For considering IC magnetic fluctuation at a relatively high energy, we have made a series of scans for $S(\mathbf{k}, \omega)$ with several energies, and the result at $x=0.06$ in $t / J=2.5$ and $t^{\prime} / t=0.15$ with $T=0.05 J$ for $\omega=0.1 J$ is shown in Fig. 5. Comparing it with Fig. 4 for the same set of parameters except for $\omega=0.05 \mathrm{~J}$, we see that at low temperatures, although the positions of the IC peaks are energy independent, the IC peaks broaden and weaken in amplitude as the energy increase, and vanish at high energies. This reflects that the excitation width increases with increasing energies, and thus leads to that the lifetime of the excitations decreasing quickly with increasing energies. The present DSSF spectrum has been used to extract the doping dependence of the incommensurability $\delta(x)$, which is defined as the deviation of the peak position from the $\mathrm{AF}$ wave vector position, and the result is plotted in Fig. 6 in comparison with the experimental result ${ }^{7}$ taken from $\mathrm{La}_{2-x} \mathrm{Sr}_{x} \mathrm{CuO}_{4}$ (inset). Our result shows that $\delta(x)$ increases progressively with the doping

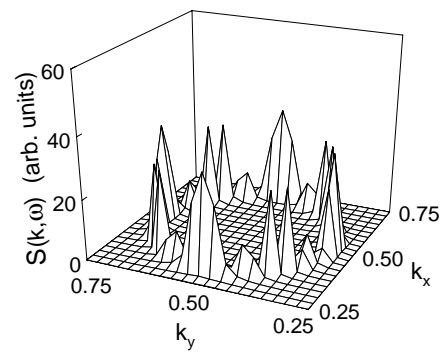

FIG. 4. The dynamical spin structure factor spectrum in the $\left(k_{x}, k_{y}\right)$ plane at $x=0.06$ in $T=0.05 J$ and $\omega=0.05 J$ with $t / J=2.5$ and $t^{\prime} / t=0.15$.

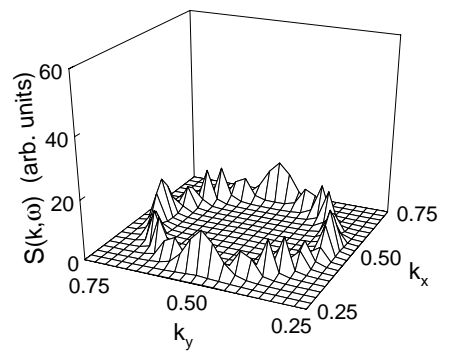

FIG. 5. The dynamical spin structure factor spectrum in the $\left(k_{x}, k_{y}\right)$ plane at $x=0.06$ with $t / J=2.5$ and $t^{\prime} / t=0.15$ in $T=0.05 J$ and $\omega=0.1 J$. concentration at lower dopings, but saturates at higher dopings, in qualitative agreement with experiments ${ }^{7,8}$.

The universal integrated dynamical spin response is a characteristic feature, and is closely related to many other normal-state properties ${ }^{1}$. The integrated dynamical spin response is manifested by the integrated dynamical spin susceptibility (IDSS), and is expressed as,

$$
I(\omega, T)=\frac{1}{N} \sum_{k} \chi^{\prime \prime}(\mathbf{k}, \omega)
$$

where the dynamical spin susceptibility is related to DSSF by the fluctuation dissipation theorem as, $\chi^{\prime \prime}(\mathbf{k}, \omega)=\left(1-e^{-\beta \omega}\right) S(\mathbf{k}, \omega)=-2 \operatorname{Im} D(\mathbf{k}, \omega)$. This IDSS has been evaluated numerically, and the results at $x=0.12$ for $t / J=2.5$ and $t^{\prime} / t=0.15$ with $T=0.2 J$ (solid line), $T=0.3 J$ (dashed line), and $T=0.5 \mathrm{~J}$ (dotted line) are plotted in Fig. 7 in comparison with the experimental data ${ }^{9}$ taken from $\mathrm{La}_{2-x} \mathrm{Sr}_{x} \mathrm{CuO}_{4}$ (inset). It is shown that the shape of IDSS appears to be particularly universal, and can be scaled approximately as $I(\omega, T) \propto \arctan \left[a_{1} \omega / T+a_{3}(\omega / T)^{3}\right]$, where $I(\omega, T)$ is almost constant for $\omega / T>1$, and then begin to decrease with decreasing $\omega / T$ for $\omega / T<1$, also in qualitative agreement with experiments ${ }^{9,10}$.

Although the scattering of the dressed spinons dominates the spin dynamics, the effect of the dressed holons

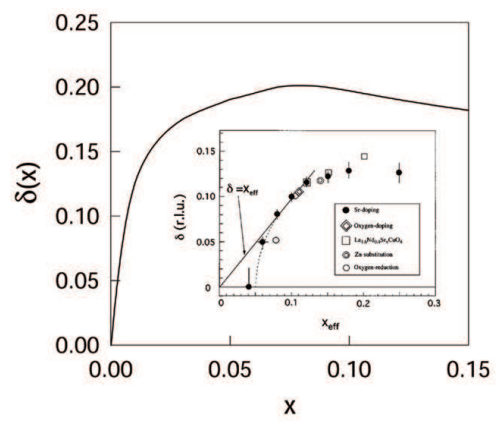

FIG. 6. The doping dependence of the incommensurability $\delta(x)$. Inset: the experimental result of $\mathrm{La}_{2-x} \mathrm{Sr}_{x} \mathrm{CuO}_{4}$ taken from Ref. [7].

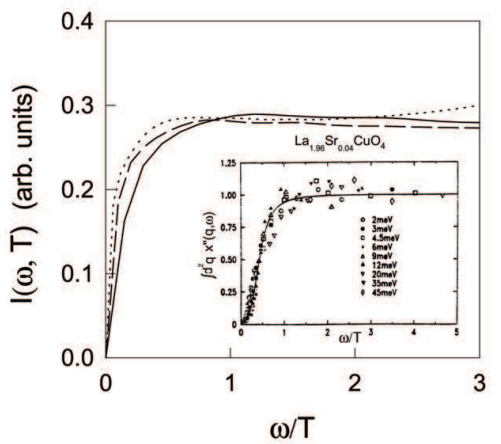

FIG. 7. The integrated dynamical spin susceptibility at $x=0.12$ with $t / J=2.5$ and $t^{\prime} / t=0.15$ in $T=0.2 J$ (solid line), $T=0.3 J$ (dashed line), and $T=0.5 J$ (dotted line). Inset: the experimental result of $\mathrm{La}_{2-x} \mathrm{Sr}_{x} \mathrm{CuO}_{4}$ taken from Ref. [9]. 
on the dressed spinon part is critical in determining the characteristic feature of the IC magnetic correlation, which can be understood from the properties of the dressed spinon excitation spectrum $E_{k}^{2}=\omega_{k}^{2}+$ $\operatorname{Re} \Sigma_{s}^{(2)}\left(\mathbf{k}, E_{k}\right)$. During the calculation of DSSF spectrum in Eq. (24), we find when $W\left(\mathbf{k}_{\delta}, \omega\right)=\left[\omega^{2}-\omega_{k_{\delta}}^{2}-\right.$ $\left.\operatorname{Re} \Sigma_{s}^{(2)}\left(\mathbf{k}_{\delta}, \omega\right)\right]^{2} \sim 0$ at some critical wave vectors $\pm \mathbf{k}_{\delta}$ in low energies, the IC peaks appear, then the weight of the IC peaks is dominated by the inverse of the imaginary part of the dressed spinon self-energy $1 / \operatorname{Im} \Sigma_{s}^{(2)}\left(\mathbf{k}_{\delta}, \omega\right)$. Thus the positions of the IC peaks are determined by both functions $W(\mathbf{k}, \omega)$ and $\operatorname{Im} \Sigma_{s}^{(2)}(\mathbf{k}, \omega)$, where the zero points of $W(\mathbf{k}, \omega)$ (then the critical wave vectors $\mathbf{k}_{\delta}$ ) is doping dependence. Near the half-filling, the zero point of $W(\mathbf{k}, \omega)$ locates at the $\mathrm{AF}$ wave vector $[1 / 2,1 / 2]$, so the commensurate AF peak appears there. With dopings, the holes disturb the AF background. Within the partial CSS framework, as a result of the self-consistent motion of the dressed holons and spinons, the IC magnetic correlation is developed beyond a certain critical doping, this reflects the fact that the low energy spin excitations drift away from the $\mathrm{AF}$ wave vector, or the zero point of $W\left(\mathbf{k}_{\delta}, \omega\right)$ is shifted from $[1 / 2,1 / 2]$ to $\mathbf{k}_{\delta}$. As seen from Eq. (24), the physics is dominated by the dressed spinon self-energy renormalization due to the dressed holon pair bubble. In this sense, the mobile dressed holons are the key factor leading to the IC magnetic correlation, i.e., the mechanism of the IC type of structure in doped cuprates is most likely related to the dressed holon motion. This is why the position of the IC peaks can be determined in the present study within the $t-t^{\prime}-J$ model, while the dressed spinon energy dependence is ascribed purely to the self-energy effects which arise from the dressed holon-spinon interaction. Since the values of $\operatorname{Im} \Sigma_{s}^{(2)}(\mathbf{k}, \omega)$ increase with increasing energies, then all values of $1 / \operatorname{Im} \Sigma_{s}^{(2)}(\mathbf{k}, \omega)$ are very small at high energies, which leads to the IC peaks disappearing at high energies.

\section{SUMMARY AND DISCUSSIONS}

In summary, we have developed a partial CSS fermionspin theory to study the physical properties of the underdoped cuprates. In this approach, the physical electron is decoupled completely as the dressed holon and spinon, with the dressed holon keeps track of the charge degree of freedom together with the phase part of the spin degree of freedom, while the dressed spinon keeps track of the amplitude part of the spin degree of freedom. The electron local constraint for single occupancy is satisfied in analytical calculations. The dressed holon is a magnetic dressing, and it behaves like a spinful fermion, while the dressed spinon is neither boson nor fermion, but a hardcore boson. Moreover, both dressed holon and spinon are gauge invariant, and in this sense, they are real and can be interpreted as physical excitations. In the common decoupling scheme, we obtain the full dressed holon and spinon Green's functions by using the equation of motion method.

Within this theoretical framework, we have studied the charge and spin dynamics of the underdoped cuprates based on the $t-t^{\prime}-J$ model. The conductivity spectrum contains a non-Drude low energy peak and a broad midinfrared band, while the temperature dependent resistivity is characterized by a crossover from the moderate temperature metallic-like to the low temperature insulatinglike behavior in the heavily underdoped regime, and a temperature linear dependence with deviations at low temperatures in the underdoped regime. The commensurate neutron scattering peak at the half-filling is split into IC peaks with dopings, where the incommensurability is doping dependent, and increases with the hole doping concentration at lower dopings, and saturates at higher dopings. These results are qualitatively similar to those seen in experiments. It is essential that these theoretical results were obtained without any adjustable parameters. These results also show that the charge dynamics is mainly governed by the scattering from the dressed holons due to the dressed spinon fluctuation, while the scattering from the dressed spinons due to the dressed holon fluctuation dominates the spin dynamics. In this case, the spin and charge dynamics in the normal-state are almost independent, and the perturbations that interact primarily with the charge do not much affect the $\operatorname{spin}^{21}$, therefore the notion of partial CSS naturally accounts for the qualitative features of the normal-state of the underdoped cuprates.

Based on this partial CSS fermion-spin theory, we have discussed the mechanism of HTSC in doped cuprates ${ }^{41}$. It is shown that dressed holons interact occurring directly through the kinetic energy by exchanging the dressed spinon excitations, leading to a net attractive force between the dressed holons, then the electron Cooper pairs originating from the dressed holon pairing state are due to the charge-spin recombination, and their condensation reveals the superconducting ground-state. The electron superconducting transition temperature is determined by the dressed holon pair transition temperature, and is proportional to the hole doping concentration in the underdoped regime. To our present understanding, the main reasons why the present theory is successful in studying the physical properties of doped cuprates are that (1) the electron local constraint is exactly obeyed during analytical calculations in contrast with the slave-particle approach, where the local constraint is explicitly replaced by a global one ${ }^{2,29}$. In this case, the representation space in the slave-particle approach is much larger than the representation space for the physical electron. (2) Since the extra $U(1)$ gauge degree of freedom related with the local constraint has been incorporated into the dressed holon, this leads to the fact that the dressed holon and spinon are gauge invariant in the partial CSS fermion-spin theory. However, the bare holon and spinon in the slave- 
particle theory are strongly coupled by the $U(1)$ gauge field fluctuation, they are not gauge invariant. (3) The representation of the dressed spinon in terms of the spin raising and lowering operators is essential in the present approach $^{29}$, because whenever a dressed holon hops it gives rise immediately to a change of the spin background as a result of careful treatment of the constraint given in Sec. II. This is why the dressed holon-spinon interaction (kinetic part) dominates the essential physics of the underdoped cuprates ${ }^{31,38}$.

\section{ACKNOWLEDGMENTS}

The authors would like to thank Professor Z.Q. Huang, Professor Z.B. Su, Professor L. Yu, Dr. F. Yuan, and Professor Z.X. Zhao for the helpful discussions. This work was supported by the National Natural Science Foundation of China under Grant Nos. 10125415 and 10074007.

${ }^{1}$ See, e.g., M.A.Kastner et al., Rev. Mod. Phys. 70, 897 (1998), and references therein.

2 See, e.g., Proceedings of Los Alamos Symposium, edited by K.S. Bedell et al. (Addison-Wesley, Redwood city, California, 1990).

${ }^{3}$ P.W. Anderson, in Frontiers and Borderlines in Many Particle Physics, edited by R.A. Broglia and J.R. Schrieffer (North-Holland, Amsterdam, 1987), p. 1; Science 235, 1196 (1987).

${ }^{4}$ See, e.g., P.W. Anderson, The Theory of Superconductivity in the High- $T_{c}$ Cuprates (Princeton, New Jersey, 1997), and references therein.

${ }^{5}$ J. Rossat-Mignod et al., Physica C 185-189, 86 (1991).

${ }^{6}$ R.J. Birgeneau et al., Phys. Rev. B 39, 2868 (1989).

${ }^{7}$ K. Yamada et al., Phys. Rev. B 57, 6165 (1998), and references therein.

${ }^{8}$ P. Dai et al., Phys. Rev. B63, 054525 (2001), and references therein.

${ }^{9}$ B. Keimer et al., Phys. Rev. B 46, 14034 (1992).

10 B.J. Sternlieb et al., Phys. Rev. B 47, 5320 (1993); R.J. Birgeneau et al., Z. Phys. B87, 15 (1992).

11 J. Orenstein et al., Phys. Rev. B 42, 6342 (1990); D.B. Tanner and T. Timusk, in Physical Properties of High Temperature Superconductors III, edited by D.M. Ginsberg (World Scientific, Singapore, 1992), p. 363, and references therein.

12 S. Uchida, Physca C 282-287, 12 (1997), and references therein.

${ }^{13}$ H. Takagi et al., Phys. Rev. Lett. 69, 2975 (1992).

${ }^{14}$ Y. Ando et al., Phys. Rev. Lett. 87, 017001 (2001); Y. Ando et al., cond-mat/0208096.

${ }^{15}$ See, e.g., Z.X. Shen and D.S. Dessau, Phys. Rep. 253, 1 (1995); A. Damascelli, Z.X. Shen, and Z. Hussain, Rev. Mod. Phys. 75, 473 (2003), and references therein.
${ }^{16}$ See, e.g., E. Dagotto, Rev. Mod. Phys. 66, 763 (1994), and references therein.

${ }^{17}$ F.D.M. Haldane, Phys. Rev. Lett. 45, 1358 (1980); Phys. Lett. 81A, 153 (1981); J. Solyom, Adv. Phys. 28, 201(1979).

${ }^{18}$ H. Yokoyama and M. Ogata, Phys. Rev. Lett. 67, 3610 (1991); F.F. Assaad and D. Würtz, Phys. Rev. B 44, 2681 (1991).

19 C. Kim et al., Phys. Rev. Lett. 77, 4054 (1996).

${ }^{20}$ See, e.g., S. Maekawa and T. Tohyama, Rep. Prog. Phys. 64, 383 (2001), and references therein.

${ }^{21}$ P.W. Anderson, Phys. Rev. Lett. 67, 2092 (1991); Science 288, 480 (2000); cond-mat/0108522.

${ }^{22}$ R.W. Hill et al., Nature 414, 711 (2001).

${ }^{23}$ N. Furukawa et al., Phys. Rev. Lett. 81, 3195 (1998); S. Maekawa and T. Tohyama, J. Phys. Chem. Solids 59, 1897 (1998).

${ }^{24}$ G.B. Martins et al., Phys. Rev. B60, R3716 (1999); G.B. Martins et al., Phys. Rev. B 63, 014414 (2000); G.B. Martins et al., Phys. Rev. Lett. 84, 5844 (2000).

${ }^{25}$ R.B. Laughlin, Phys. Rev. Lett. 79, 1726 (1997); J. Low. Tem. Phys. 99, 443 (1995).

${ }^{26}$ F.C. Zhang and T.M. Rice, Phys. Rev. B37, 3759 (1988).

27 T.M. Rice, Physica C282-287, xix (1997), and references therein.

${ }^{28}$ C. Gros, R. Joynt, and T.M. Rice, Phys. Rev. B 36, 381 (1987).

29 Shiping Feng, Z.B. Su, and L. Yu, Phys. Rev. B 49, 2368 (1994); Mod. Phys. Lett. B7, 1013 (1993).

30 P.B. Wiegmann, Phys. Rev. Lett. 60, 821 (1988).

${ }^{31}$ Shiping Feng and Feng Yuan, in Symposium on the Frontiers of Physics at Millennium, edited by Y.L. Wu and J.P. Hsu (World Scientific, Singapore, 2001), p. 221.; Shiping Feng, Feng Yuan, Weiqiang Yu, and Pengpeng Zhang, Phys. Rev. B 60, 7565 (1999).

${ }^{32}$ N.M. Plakida, cond-mat/0210385.

${ }^{33}$ D.N. Zubarev, Sov. Phys. Usp. 3, 201 (1960).

${ }^{34}$ B. Keimer et al., Phys. Rev. B 46, 14034 (1992); M. Matsuda et al., Phys. Rev. B 62, 9148 (2000).

${ }^{35}$ Shiping Feng and Yun Song, Phys. Rev. B 55, 642 (1997).

36 J. Kondo and K. Yamaji, Prog. Theor. Phys. 47, 807 (1972).

37 See, e.g., S.V. Tyablikov, Method in the Quantum Theory of Magnetism (Plenum, New York, 1967).

38 Shiping Feng and Zhongbing Huang, Phys. Rev. B 57, 10328 (1998); Feng Yuan, Shiping Feng, Zhao-Bin Su, and Lu Yu, Phys. Rev. B 64, 224505 (2001).

39 J. Zaanen and O. Gunnarsson, Phys. Rev. B 40, 7391 (1989); D. Poilblanc and T.M. Rice, Phys. Rev. B 39, 9749 (1989); H.J. Schulz, Phys. Rev. Lett. 64, 1445 (1990); N. Bulut, D. Hone, D.J. Scalapino, and N.E. Bickers, Phys. Rev. Lett 64, 2723 (1990); T. Tanamoto, H. Kohno, and H. Fukuyama, J. Phys. Soc. Jpn. 63, 2739 (1994); Y. Hasegawa and H. Fukuyama, Japanese J. App. Phys. 26, L322 (1987).

${ }^{40}$ H.A. Mook et al., Phys. Rev. Lett. 70, 3490 (1993); H.F. Fong et al., Nature 398, 588 (1999); H. He et al., Science 295, 1045 (2002).

${ }^{41}$ Shiping Feng, Phys. Rev. B 68, 184501 (2003). 